\title{
Contour changes in human alveolar bone following tooth extraction of the maxillary central incisor
}

\author{
Bei LI, Yao WANG \\ (Department of Stomatology, Civil Aviation General Hospital, Beijing 100123, China) \\ E-mail: bbpretty1219@163.com; 981947317@qq.com \\ Received Apr. 28, 2014; Revision accepted Oct. 9, 2014; Crosschecked Nov. 23, 2014
}

\begin{abstract}
The purpose of this study was to apply cone-beam computed tomography (CBCT) to observe contour changes in human alveolar bone after tooth extraction of the maxillary central incisor and to provide original morphological evidence for aesthetic implant treatment in the maxillary anterior area. Forty patients were recruited into the study. Each patient had two CBCT scans (CBCT I and CBCT II), one taken before and one taken three months after tooth extraction of maxillary central incisor (test tooth $\mathrm{T}$ ). A fixed anatomic reference point was used to orient the starting axial slice of the two scans. On three CBCT I axial slices, which represented the deep, middle, and shallow layers of the socket, labial and palatal alveolar bone widths of T were measured. The number of sagittal slices from the start point to the pulp centre of T was recorded. On three CBCT II axial slices, the pulp centres of extracted T were oriented according to the number of moved sagittal slices recorded in СBCT I. Labial and palatal alveolar bone widths at the oriented sites were measured. On the СBCT I axial slice which represented the middle layer of the socket, sagittal slices were reconstructed. Relevant distances of T on the sagittal slice were measured, as were the alveolar bone width and tooth length of the opposite central incisor. On the CBCT II axial slice, which represented the middle layer of the socket, relevant distances recorded in CBCT I were transferred on the sagittal slice. The height reduction of alveolar bone on labial and palatal sides was measured, as were the alveolar bone width and tooth length of the opposite central incisor at the oriented site. Intraobserver reliability assessed by intraclass correlation coefficients (ICCs) was high. Paired sample $t$-tests were performed. The alveolar bone width and tooth length of the opposite central incisor showed no statistical differences $(P<0.05)$. The labial alveolar bone widths of $\mathrm{T}$ at the deep, middle, and shallow layers all showed statistical differences. However, no palatal alveolar bone widths showed any statistical differences. The width reduction of alveolar bone was 1.2, 1.6, and $2.7 \mathrm{~mm}$ at the deep, middle, and shallow layers, respectively. The height reduction of alveolar bone on labial and palatal sides of T both showed statistical differences, which was 1.9 and $1.1 \mathrm{~mm}$, respectively.
\end{abstract}

Key words: Cone-beam computed tomography, Alveolar bone contour, Maxillary central incisor doi:10.1631/jzus.B1400119

Document code: A

CLC number: R782

\section{Introduction}

Esthetic outcome is becoming a more essential factor than functional restoration for patients with anterior dentition defect. The physiological remodelling of alveolar bone becomes very active and com-

\footnotetext{
${ }^{\ddagger}$ Corresponding author

(6) ORCID: Yao WANG, http://orcid.org/0000-0003-3386-0317

(C) Zhejiang University and Springer-Verlag Berlin Heidelberg 2014
}

plex after tooth extraction. Generally, concurrent with new bone growth into the extraction socket, there is also resorption of the alveolar bone. Many studies have demonstrated that the height and especially the width of the alveolar bone decrease significantly after tooth extraction (Lam, 1960; Pietrokovski and Massler, 1967; Johnson, 1969; Jahangiri et al., 1998; Schropp et al., 2003; Araujo and Lindhe, 2005; Kerr et al., 2008; van der Weijden et al., 2009), leading to insufficient bone volume at the implant site. 
Cone-beam computed tomography $(\mathrm{CBCT})$ is a new technique with advantages of rapid scan speed, high resolution, low effective radiation dose (Cohnen et al., 2002; Ngan et al., 2003; Schulze et al., 2004), and high radiation efficiency. It is ideal for clinical applications (Hatcher et al., 2003; Vannier, 2003). It reduces the slice thickness of each image to tens of micrometers, which is indispensable for precisely observing the fine structure of the oral and maxillofacial region (Loubele et al., 2007). CBCT generates volumetric data via scanning. Various flat or curved planes, on which quantitative measurements are made, can be easily accessed by image reconstruction. Although $\mathrm{CBCT}$ has many advantages, there are few studies using $\mathrm{CBCT}$ to measure horizontal and vertical bone resorption following tooth extraction. In this study, we use CBCT to observe contour changes of human alveolar bone in the esthetic area of maxillary central incisor, and provide original morphological evidence for esthetic implant treatment.

\section{Materials and methods}

\subsection{Population and criteria}

Forty patients ( 20 males and 20 females), with ages between 20 and 63 years old, were recruited from people who needed an extraction of the maxillary central incisor and who wanted to restore the dentition defect with a dental implant.

The inclusion criteria were: (1) The patients had a maxillary central incisor to be extracted because of injury or endodontic or periapical disease. Each patient had two CBCT scans. One was taken an hour before tooth extraction, and the other was taken three months after extraction. (2) All extraction and examinations were performed by one clinician. Two clinical examinations at the time of tooth extraction and three months after tooth extraction showed normal plaque index, bleeding index, and probing depth of the patients. (3) Adjacent teeth to the test tooth $\mathrm{T}$ received no treatment in the interval between two CBCT scans. On two CBCT scans, at least two-thirds of the roots of the adjacent teeth were surrounded by bone.

The exclusion criteria were: (1) The first CBCT scan showed congenital defects like bone fenestration or dehiscence on the bone wall. (2) Fractures due to extraction were found on the bone wall with the examination of periodontal probe. (3) The patients had untreated periodontal disease with the symptom of swollen gums, dental calculus, loose teeth, bleeding on probing, purulent exudates. (4) The patients had diabetes or thyroid disease or other diseases that might influence bone remodelling. (5) The patients had drug therapy of estrogens or anticoagulants. (6) The patients had smoking history and could not quit. (7) Women patients during pregnancy and lactation.

The procedure of extraction of the maxillary central incisor was carried out as follows: patients were anesthetized with local infiltration of $2 \%(0.02 \mathrm{~g} / \mathrm{ml})$ lidocaine which contained epinephrine at 1:100000 $(\mathrm{v} / \mathrm{v})$. Dental elevators and forceps were used to carefully extract the teeth without any flap elevation. Granulation tissues were removed by curettes. Extraction sockets were examined with a periodontal probe. Patients were prescribed mouth rinses postoperatively with $0.2 \%(2 \mathrm{~g} / \mathrm{L})$ chlorhexidine, twice per day. Oral analgesics with ibuprofen $800 \mathrm{mg}$ t.i.d. was administered as needed.

\subsection{CBCT equipment and analysis software}

NewTom VG (Quantitative Radiology, Verona, Italy); High resolution mode; Voxel, $0.125 \mathrm{~mm}$; $\mathrm{X}$-ray settings, $110 \mathrm{kV}$ and 1-20 mA; field of view (FOV) size, $12 \mathrm{~cm} \times 8 \mathrm{~cm}$; NNT VIEWER 2.19 (Slice settings: width $120 \mathrm{~mm}$, thickness $0.125 \mathrm{~mm}$, distance $0.125 \mathrm{~mm}$ ).

\subsection{Research methods}

The first CBCT the patient received was referred to as CBCT I, and the second CBCT II. The maxillary central incisor, which was to be extracted in CBCT I and was extracted in CBCT II, was referred to as test tooth T. On any CBCT I axial slice that was in the middle third section of the root of the lateral incisor, sagittal slices were reconstructed to measure its root length (Fig. 1). The root apex of the lateral incisor next to $\mathrm{T}$ was used to orient the starting axial slice Ax R on the two CBCT scans (Fig. 2).

The axial slices, which were at a depth of $20 \%$, $50 \%$, and $70 \%$ of the root length of the lateral incisor, were calculated and referred to as Ax20, Ax50, and Ax70 to represent the deep, middle, and shallow layers of the socket, respectively. On CBCT I, Ax20, Ax50, 
and Ax70 sagittal slices were reconstructed based on the line connecting two pulp centres of the lateral incisor and the opposite central incisor. Alveolar bone was divided by the line into two parts, labial alveolar bone and palatal alveolar bone.

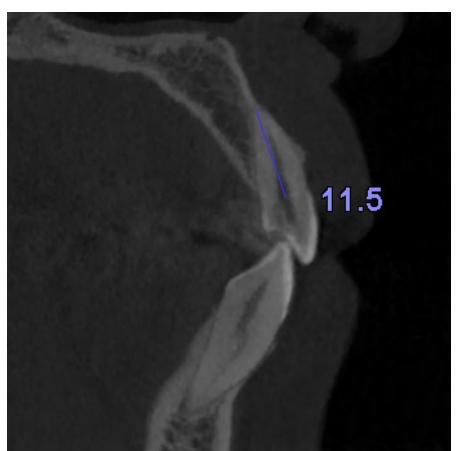

Fig. 1 Tooth length of lateral incisor in CBCT I
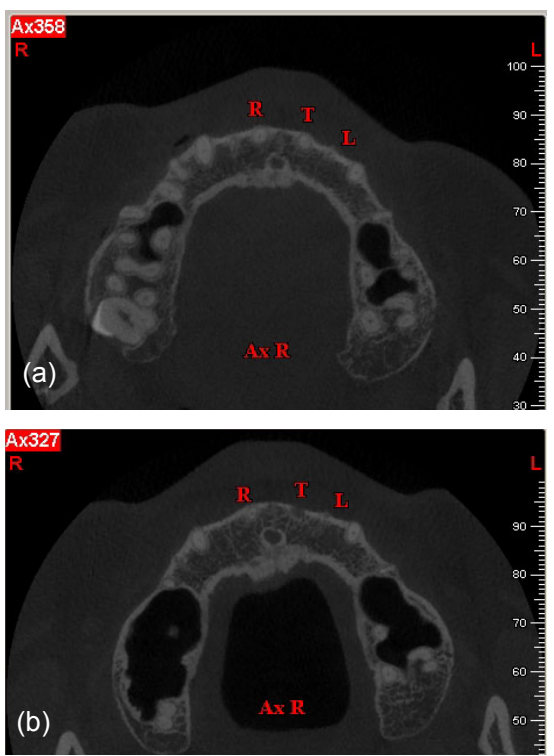

Fig. 2 Root apex of the lateral incisor to orient $A x R$ in CBCT I (a) and CBCT II (b)

The starting sagittal slice that passed through the pulp centre of the lateral incisor was referred to as $\mathrm{Cx}$ R. The sagittal slice that passed through the pulp centre of the test tooth $\mathrm{T}$ on $\mathrm{Ax} 20, \mathrm{Ax} 50$, and $\mathrm{Ax} 70$ was referred to as $\mathrm{Cx} \mathrm{T}-20, \mathrm{Cx} \mathrm{T}-50$, and $\mathrm{Cx} \mathrm{T}-70$, respectively. The number of sagittal slices from $\mathrm{Cx} \mathrm{R}$ to $\mathrm{Cx} \mathrm{T}-20$, to $\mathrm{Cx} \mathrm{T}-50$, to $\mathrm{Cx} \mathrm{T}-70$ was recorded as N1-20, N1-50, and N1-70, respectively. Labial and palatal alveolar bone widths of $\mathrm{T}$ were measured at the sites of Cx T-20 on Ax20, Cx T-50 on Ax50, and Cx T-70 on Ax70, respectively (Fig. 3).
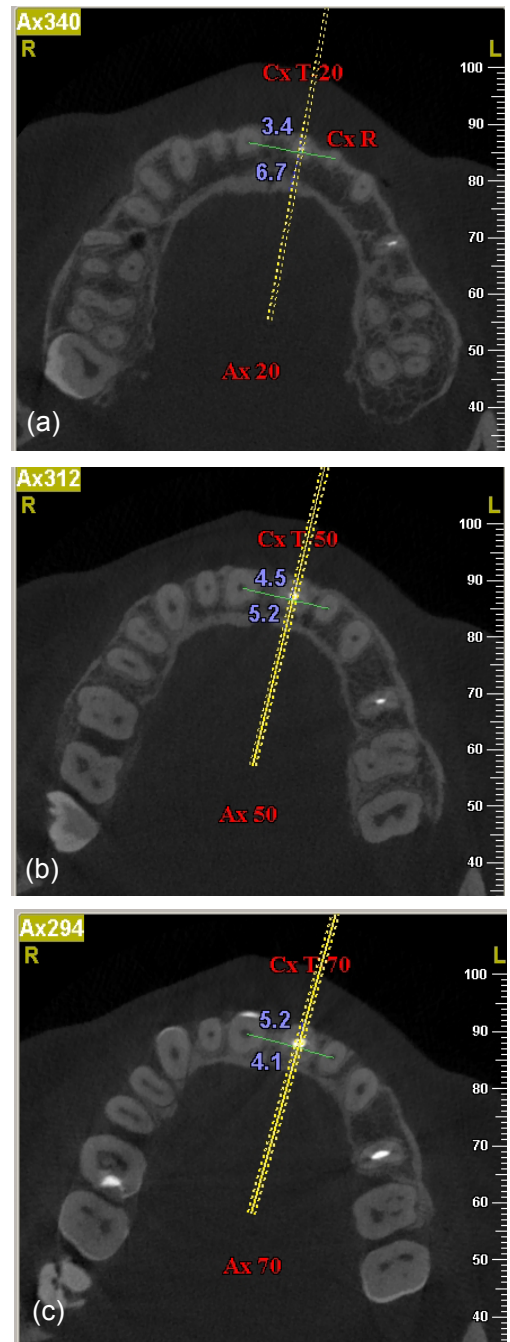

Fig. 3 Labial and palatal alveolar bone widths of $T$ at Cx T-20 on Ax20 (a), Cx T-50 on Ax50 (b), and Cx T-70 on $\mathbf{A x 7 0}(c)$ in CBCT I

On sagittal slice Cx T-50, a line was drawn between two spines to set the palatal plane. The distal spine was abbreviated to $\mathrm{D}$. The apices of the labial and palatal alveolar ridges were designated $\mathrm{A}$ and $\mathrm{B}$. Perpendiculars to the palate plane passing through A and $\mathrm{B}$ were drawn. The intersection points were named L and P. Distances of AL, BP, DL, and DP were measured (Fig. 4). The sagittal slice that passed through the pulp centre of the opposite central incisor on Ax50 was designated Cx F. The number of sagittal slices from $\mathrm{Cx} \mathrm{R}$ to $\mathrm{Cx} \mathrm{F}$ was recorded as N2. Alveolar 
bone width and tooth length of the opposite central incisor were measured on Ax50 and Cx F (Figs. 5 and 6).

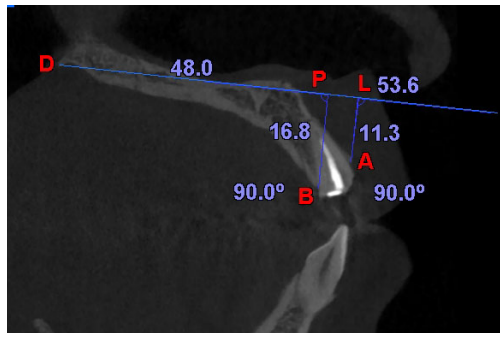

Fig. 4 Relevant distances of T on Cx T-50 in CBCT I

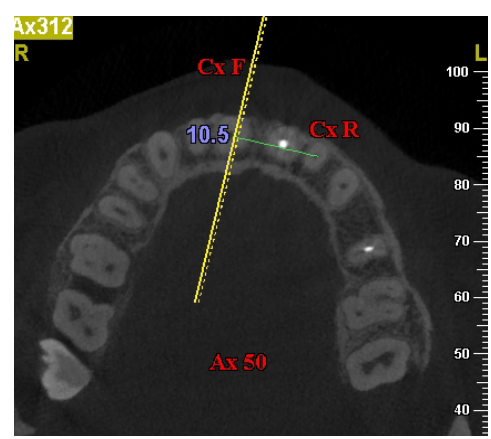

Fig. 5 Alveolar bone width of the opposite central incisor at $\mathrm{Cx} F$ on $\mathrm{Ax50}$ in $\mathrm{CBCT} I$

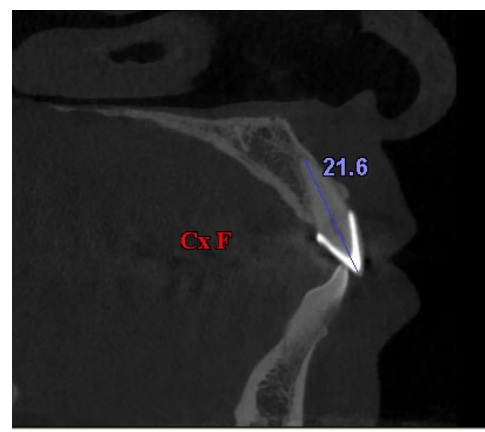

Fig. 6 Tooth length of the opposite central incisor on CX F in CBCT I

Among CBCT II slice series, the slice was first oriented to Ax R (Fig. 2b). On CBCT II Ax20, Ax50, and $\mathrm{Ax} 70$, sagittal slices were reconstructed as in CBCT I. The same numbers of sagittal slices N1-20, $\mathrm{N} 1-50$, and N1-70 were taken from $\mathrm{Cx}$ R to orient extracted $\mathrm{T}$, respectively. Labial and palatal alveolar bone widths of $\mathrm{T}$ were measured at the oriented sites on Ax20, Ax50, and Ax70 (Fig. 7).

On N1-50-oriented sagittal slice, distances of $\mathrm{AL}, \mathrm{BP}, \mathrm{DL}$, and DP were transferred to measure the labial and palatal height reductions of alveolar bone along the original root contour (Fig. 8). The same number of sagittal slices N2 was taken from $\mathrm{Cx}$ R to orient the opposite central incisor. Alveolar bone width and tooth length of the opposite central incisor were measured on Ax50 and N2-oriented sagittal slice (Figs. 9 and 10).
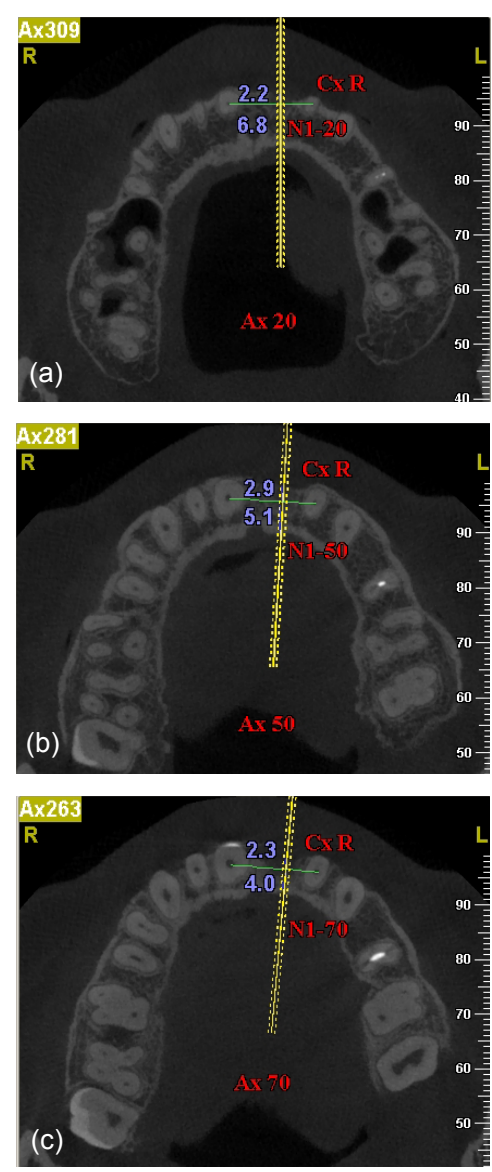

Fig. 7 Labial and palatal alveolar bone widths of $T$ at N1-20-oriented site on Ax20 (a), N1-50-oriented site on Ax50 (b), and N1-70-oriented site on $\mathbf{A x 7 0}$ (c) in CBCT II

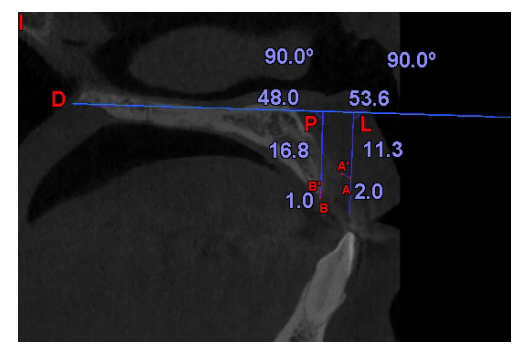

Fig. 8 Relevant distances of $\mathrm{T}$ transferred on N1-50oriented sagittal slice in CBCT II 


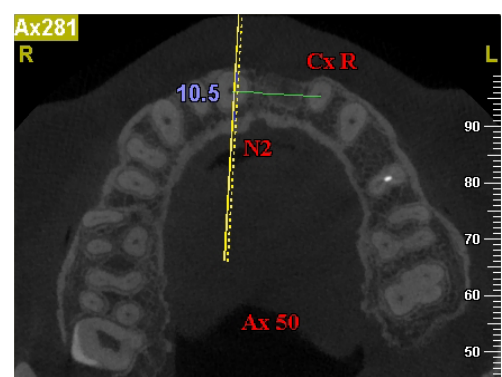

Fig. 9 Alveolar bone width of the opposite central incisor at N2-oriented site on Ax50 in CBCT II

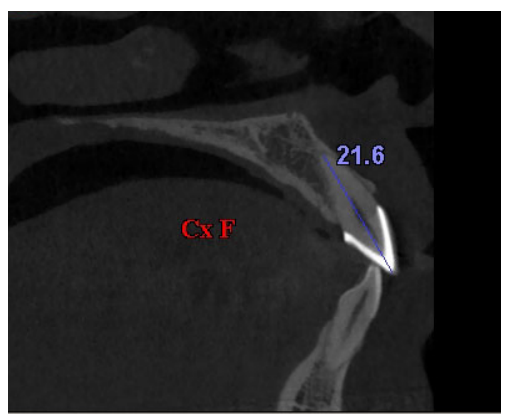

Fig. 10 Tooth length of the opposite central incisor on N2-oriented slice in CBCT II

\subsection{Statistical analysis}

All measurements on CBCT scans were performed by one investigator. To test the intraobserver reliability, 5 patients were randomly selected from the 40 patients. Only CBCT I was used. Orientations of Ax R of each patient were repeated. Sagittal slices were reconstructed based on the line connecting two pulp centres of the lateral incisor and the opposite central incisor on Ax50. Measurements of alveolar bone width on Ax50 and tooth length on Cx T-50 of T were repeated. Orientations and measurements were carried out on $2 \mathrm{~d}$, one week apart and five times each day. Intraclass correlation coefficients (ICCs) for absolute agreement based on a one-way random effects analysis of variance (ANOVA) were calculated using SPSS 18.0 (SPSS Inc., Chicago, USA) software. Means and standard deviations (SDs) of alveolar bone width, tooth length, and height reduction were calculated using SPSS. Paired sample $t$-tests were performed. A $P$ value $<0.05$ was selected to indicate a significant difference.

\section{Results and discussion}

The ICC values for the orientations of Ax R, measurements of alveolar bone width and tooth length were $0.997,0.991$, and 0.995 , respectively. Tooth length and alveolar bone width of the opposite central incisor measured from two CBCT scans are shown in Table 1. Paired sample $t$-tests of both measurements showed no statistically significant differences.

Table 1 Tooth length and alveolar bone width of the opposite central incisor measured from two CBCT scans

\begin{tabular}{ccc}
\hline Scan & $\begin{array}{c}\text { Tooth length } \\
(\mathrm{mm})\end{array}$ & $\begin{array}{c}\text { Alveolar bone width } \\
(\mathrm{mm})\end{array}$ \\
\hline CBCT I & $21.1 \pm 1.3$ & $11.9 \pm 1.6$ \\
CBCT II & $21.1 \pm 1.3$ & $11.9 \pm 1.6$ \\
\hline$P$ & 0.71 & 0.39 \\
\hline
\end{tabular}

Data are expressed as mean $\pm \mathrm{SD}, n=40$

Labial, palatal, and total alveolar bone widths of $\mathrm{T}$ on Ax20, Ax50, and Ax70 measured from two CBCT scans are shown in Table 2. Paired sample $t$-tests of width measurements of labial and total alveolar bone on $\mathrm{Ax} 20, \mathrm{Ax} 50$, and $\mathrm{Ax} 70$ all showed statistically significant differences, while width measurements of palatal alveolar bone all showed no statistical differences. The average width reduction of labial and total alveolar bone was $2.7 \mathrm{~mm}$ on Ax70, $1.6 \mathrm{~mm}$ on $\mathrm{Ax} 50$, and $1.2 \mathrm{~mm}$ on Ax20, respectively.

Table 2 Labial, palatal, and total alveolar bone widths of $\mathrm{T}$ on Ax20, Ax50, and Ax70 measured from two CBCT scans

\begin{tabular}{|c|c|c|c|c|c|c|c|c|c|}
\hline \multirow{2}{*}{ Scan } & \multicolumn{3}{|c|}{ Labial width (mm) } & \multicolumn{3}{|c|}{ Palatal width (mm) } & \multicolumn{3}{|c|}{ Total width (mm) } \\
\hline & Ax20 & Ax 50 & $\mathrm{Ax} 70$ & $\mathrm{Ax} 20$ & Ax50 & Ax70 & Ax20 & Ax50 & Ax70 \\
\hline CBCT I & $4.2 \pm 0.2$ & $5.3 \pm 0.2$ & $4.8 \pm 0.3$ & $8.3 \pm 0.7$ & $5.8 \pm 0.2$ & $4.5 \pm 0.4$ & $12.7 \pm 0.6$ & $11.1 \pm 0.7$ & $9.3 \pm 0.3$ \\
\hline CBCT II & $3.0 \pm 0.3$ & $3.7 \pm 0.4$ & $2.1 \pm 0.2$ & $8.3 \pm 0.7$ & $5.8 \pm 0.2$ & $4.5 \pm 0.4$ & $11.5 \pm 0.4$ & $9.6 \pm 0.5$ & $6.6 \pm 0.3$ \\
\hline Difference & $1.2 \pm 0.3$ & $1.6 \pm 0.3$ & $2.7 \pm 0.1$ & $0.0 \pm 0.0$ & $0.0 \pm 0.0$ & $0.0 \pm 0.0$ & $1.2 \pm 0.6$ & $1.5 \pm 0.5$ & $2.7 \pm 0.2$ \\
\hline
\end{tabular}


Paired sample $t$-tests of measurements of height reduction of labial and palatal alveolar bone both showed statistically significant differences $(P<0.05)$ The average height reductions of labial and palatal alveolar bones were $(1.9 \pm 0.6)$ and $(1.1 \pm 0.2) \mathrm{mm}$, respectively.

In this study, the comparison of tooth length and alveolar bone width of the opposite central incisor has verified the feasibility of using CBCT to continuously observe contour changes in human alveolar bone at different time points. Tooth length was measured on the sagittal slice. The comparison of tooth length verified the accuracy of sagittal orientation. Alveolar bone width was measured on the axial slice, while the site for measurement was also determined by the oriented sagittal slice. The comparison of alveolar bone width verified the accuracy of both axial and sagittal orientations. In conclusion, by referencing a fixed anatomic point, the same axial slice from different CBCT scans of one patient could be found. By moving the same number of sagittal slices as originally recorded, the same sagittal slice could be found. The orientation method makes measurements comparable in a scientific way.

According to the statistics in Xie (2005), the average root lengths of the maxillary central and lateral incisor were 11.3 and $11.5 \mathrm{~mm}$, respectively. Theoretically, axial slices of the lateral incisor include all those of the central incisor. Root apex of the lateral incisor was set as a reference point to orient the starting axial slice of different CBCT scans from one patient. Three layers, each from the deep, middle, and shallow third sections of the root of the lateral incisor, were chosen to observe contour changes of human alveolar bone in the esthetic area of the maxillary central incisor. The shallow layer was set near the middle third section of the root, considering the vertical resorption of alveolar bone after tooth extraction.

After three-month extraction of the maxillary central incisor, horizontal resorption of labial alveolar bone occurred at different depths of the extraction site, and the further towards the alveolar ridge, the more horizontal resorption occurred. In this study, there was an average width reduction of $2.7 \mathrm{~mm}$ near the alveolar ridge, which is less than that reported in most studies. Some clinical studies (Lekovic et al., 1998;
Camargo et al., 2000) performed flap elevation and placed titanium pins in the labial alveolar bone of patients as markers at the time of tooth extraction. After six months of healing, a full-thickness flap was elevated again to measure the width and height of the alveolar ridge at the titanium pins. Lekovic et al. (1998) reported an average width reduction of $4.6 \mathrm{~mm}$, while Camargo et al. (2000) reported $3 \mathrm{~mm}$. A clinical study of Schropp et al. (2003) reported that the width of the alveolar ridge reduced by $50 \%$ at 12 months after tooth extraction and the bone loss was between 5 and $7 \mathrm{~mm}$. van der Weijden et al. (2009) conducted a systematic review and concluded that the reduction in width of the alveolar ridge was $3.87 \mathrm{~mm}$.

One of the possible explanations for the inconsistent amount of horizontal bone resorption was that the observation interval was different. The observation interval of this study was three months, shorter than those of the abovementioned studies. Since the alveolar bone resorption continues throughout life (Jahangiri et al., 1998), the longer the interval, the greater will be the amount of bone resorption. In addition, it was well established that the elevation of a full-thickness flap might cause crestal alveolar bone loss of about $0.6 \mathrm{~mm}$ (Wood et al., 1972), making tooth extraction no longer the sole factor causing alveolar bone resorption (Fickl et al., 2008). This might partially be the reason why studies using the method of flap elevation (Lekovic et al., 1998; Camargo et al., 2000) reported more resorption. Despite the inconsistent amount of bone resorption, most studies agreed the fact that the horizontal reduction was more pronounced at the labial bone wall than at the palatal bone wall (Lekovic et al., 1998; Schropp et al., 2003; Araujo and Lindhe, 2005; Barone et al., 2008).

This study has also proved that after threemonth extraction of the maxillary central incisor, vertical resorption occurred on both labial and palatal alveolar bones. The height reduction of the labial plate was $1.9 \mathrm{~mm}$, and that of the palatal plate $1.1 \mathrm{~mm}$. Lekovic et al. (1998) reported an average buccal height reduction of $1.5 \mathrm{~mm}$, Camargo et al. (2000) reported $1 \mathrm{~mm}$, and van der Weijden et al. (2009) reported 1.67-2.03 mm. Most studies were consistent with the conclusion that the height reduction was 
more pronounced at the labial bone wall than at the palatal bone wall (Lekovic et al., 1998; Schropp et al., 2003; Araujo and Lindhe, 2005; Barone et al., 2008).

CBCT is an ideal imaging tool to observe contour changes in human alveolar bone (Li et al., 2014). Studies of whether alveolar bone preservation techniques or materials are effective in human are suggested to make full use of CBCT.

\section{Acknowledgements}

We sincerely thank Dr. Shi-hai HE (Department of Stomatology, Civil Aviation General Hospital, Beijing, China) for encouragement and assistance for this study.

\section{Compliance with ethics guidelines}

Bei LI and Yao WANG declare that they have no conflict of interest.

All procedures followed were in accordance with the ethical standards of the responsible committee on human experimentation (institutional and national) and with the Helsinki Declaration of 1975, as revised in 2008 (5). Informed consent was obtained from all patients for being included in the study. Additional informed consent was obtained from all patients for which identifying information is included in this article.

\section{References}

Araujo, M.G., Lindhe, J., 2005. Dimensional ridge alterations following tooth extraction: an experimental study in the dog. J. Clin. Periodontol., 32(2):212-218. [doi:10.1111/j. 1600-051X.2005.00642.x]

Barone, A., Aldini, N.N., Fini, M., et al., 2008. Xenograft versus extraction alone for ridge preservation after tooth removal: a clinical and histomorphometric study. J. Periodontol., 79(8):1370-1377. [doi:10.1902/jop.2008.070628]

Camargo, P.M., Lekovic, V., Weinlaender, M., et al., 2000. Influence of bioactive glass on changes in alveolar process dimensions after exodontia. Oral Surg. Oral Med. Oral Pathol. Oral Radiol. Endodontol., 90(5):581-586. [doi:10.1067/moe.2000.110035]

Cohnen, M., Kemper, J., Mobes, O., et al., 2002. Radiation dose in dental radiology. Eur. Radiol., 12(3):634-637. [doi:10.1007/s003300100928]

Fickl, S., Zuhr, O., Wachtel, H., et al., 2008. Tissue alterations after tooth extraction with and without surgical trauma: a volumetric study in the beagle dog. J. Clin. Periodontol., 35(4):356-363. [doi:10.1111/j.1600-051X.2008.01209.x]

Hatcher, D.C., Dial, C., Mayorga, C., 2003. Cone beam CT for pre-surgical assessment of implant sites. J. Calif. Dent. Assoc., 31(11):825-833.

Jahangiri, L., Devlin, H., Ting, K., et al., 1998. Current perspectives in residual ridge remodeling and its clinical implications: a review. J. Prosthet. Dent., 80(2):224-237. [doi:10.1016/S0022-3913(98)70116-7]

Johnson, K., 1969. A study of the dimensional changes occurring in the maxilla following tooth extraction. Aust. Dent. J., 14(4):241-244. [doi:10.1111/j.1834-7819.1969. tb06001.x]

Kerr, E.N., Mealey, B.L., Noujeim, M.E., et al., 2008. The effect of ultrasound on bone dimensional changes following extraction: a pilot study. J. Periodontol., 79(2): 283-290. [doi:10.1902/jop.2008.070289]

Lam, R.V., 1960. Contour changes of the alveolar processes following extractions. J. Prosthet. Dent., 10(1):25-32. [doi:10.1016/0022-3913(60)90083-4]

Lekovic, V., Camargo, P.M., Klokkevold, P.R., et al., 1998. Preservation of alveolar bone in extraction sockets using bioabsorbable membranes. J. Periodontol., 69(9):1044-1049. [doi:10.1902/jop.1998.69.9.1044]

Li, B., Wang, Y., Li, J., 2014. A feasibility study of applying cone-beam computed tomography to observe dimensional changes in human alveolar bone. J. Zhejiang Univ.-Sci. B (Biomed. \& Biotechnol.), 15(4):393-398. [doi:10.1631/ jzus.B1300249]

Loubele, M., Guerrero, M.E., Jacobs, R., et al., 2007. A comparison of jaw dimensional and quality assessments of bone characteristics with cone beam CT, spiral tomography and multi slice spiral CT. Int. J. Oral Maxillofac. Implants, 22(3):446-454.

Ngan, D.C., Kharbanda, O.P., Geenty, J.P., et al., 2003. Comparison of radiation levels from computed tomography and conventional dental radiographs. Aust. Orthod. $J ., 19(2): 67-75$.

Pietrokovski, J., Massler, M., 1967. Alveolar ridge resorption following tooth extraction. J. Prosthet. Dent., 17(1):21-27. [doi:10.1016/0022-3913(67)90046-7]

Schropp, L., Wenzel, A., Kostopoulos, L., et al., 2003. Bone healing and soft tissue contour changes following singletooth extraction: a clinical and radiographic 12-month prospective study. Int. J. Periodontics Restorative Dent., 23(4):313-323.

Schulze, D., Heiland, M., Thurmann, H., et al., 2004. Radiation exposure during midfacial imaging using 4- and 16-slice computed tomography, cone beam computed tomography systems and conventional radiography. Dentomaxillofac. Radiol., 33(2):83-86. [doi:10.1259/dmfr/ 28403350]

van der Weijden, F., Dell'Acqua, F., Slot, D.E., 2009. Alveolar bone dimensional changes of post-extraction sockets in humans: a systematic review. J. Clin. Periodontol., 36(12): 1048-1058. [doi:10.1111/j.1600-051X.2009.01482.x]

Vannier, M.W., 2003. Craniofacial computed tomography scanning: technology, applications and future trends. Orthod. Craniofac. Res., 6(s1):23-30. [doi:10.1034/j. 1600-0544.2003.232.x]

Wood, D.L., Hoag, P.M., Donnenfeld, O.W., et al., 1972. 
Alveolar crest reduction following full and partial thickness flaps. J. Periodontol., 43(3):141-144. [doi:10.1902/ jop.1972.43.3.141]
Xie, Q.F., 2005. Dental Anatomy and Oral Physiology. Beijing University Medical Publishing House, Beijing, China, p.101 (in Chinese).

\section{中文㔍要：}

本文题目: 人类上领中切牙拔除后牙槽骨外形变化的研究

Contour changes in human alveolar bone following tooth extraction of the maxillary central incisor

研究目的：观察人类上领中切牙拔除后牙槽骨外形改建的规律。

创新要点: 锥形束计算机断层摄影 (CBCT) 在口腔硬组织测量方面具有可靠性, 在不同时期拍摄的影 像中测量具有可重复性。本研究充分利用这一影像学工具观察人类上领中切牙拔除后牙槽 骨改建的规律。

研究方法：选择 40 名患者上领中切牙拔牙前和拔牙后三个月时拍摄的两份 $\mathrm{CBCT}$ 影像资料。在第一次 $\mathrm{CBCT}$ 中, 选取中切牙拔牙窝深、中、浅三个水平断层进行矢状断层重建, 分别记录矢状断 层起点到拟拔除中切牙 (实验牙 $\mathrm{T}$ ) 牙髓中心移动的层数, 并测量实验牙 $\mathrm{T}$ 处牙槽骨宽度, 在中切牙拔牙窝中层测量腭平面相关垂直距离。在第二次 $\mathrm{CBCT}$ 的三个水平断层上, 依据 第一次记录的移动层数定位实验牙 $\mathrm{T}$, 并在定位处测量牙槽骨宽度。在中切牙拔牙窝中层上 转移第一次 CBCT 记录的腭平面相关距离, 并测量唇腭侧牙槽嵴顶沿原牙根外形垂直吸收 的距离。

重要结论: 上领中切牙拔除后三个月, 在拔牙窝的不同深度, 唇侧的牙槽骨均有不同程度的水平骨吸 收, 越接近牙槽嵴顶处牙槽骨吸收越明显。唇腭侧牙槽嵴顶均有明显的垂直骨吸收, 唇侧 牙槽嵴顶的垂直骨吸收比腭侧明显。

关键词组：锥形束计算机断层摄影 (CBCT) ; 牙槽突外形; 上领中切牙 